\title{
ON A SUBLATTICE OF THE LATTICE OF CONGRUENCES ON A SIMPLE REGULAR $\omega$-SEMIGROUP
}

\author{
G. R. BAIRD ${ }^{1}$ \\ (Received 23 September 1969) \\ Communicated by G. B. Preston
}

The set $E$ of idempotents of a semigroup $S$ can be partially ordered by defining $e \leqq f$ if and only if $e f=f e=e(e, f \in E)$. If $E=\left\{e_{i}: i=i=0,1, \cdots\right\}$ and under this ordering

$$
e_{0}>e_{1}>e_{2} \cdots,
$$

then we call $S$ an $\omega$-semigroup. Munn [10] has given a complete classification of simple regular $\omega$-semigroups in terms of groups and group homomorphisms. Let $\Lambda_{0}(S)$ denote the set of congruences on a simple regular $\omega$-semigroup $S$ consisting of those congruences which either are idempotent-separating or are group congruences on $S$. It is evident that $\Lambda_{0}(S)$ is a sublattice of the lattice of all congruences on $S$.

In this paper we determine a necessary and sufficient condition for the sublattice $\Lambda_{0}(S)$ to be modular.

If we further restrict $S$ and insist that it be bisimple then $\Lambda_{0}(S)$ becomes the full lattice of congruences on $S$ (Munn [9]). For this case Munn [9] has determined a necessary and sufficient condition for $\Lambda_{0}(S)$ to be modular. Our work generalizes Munn's theorem from bisimple $\omega$-semigroups to simple regular $\omega$-semigroups. Many of the results in this paper are straightforward generalizations of results given by Munn for the bisimple case. Whenever possible Munn's results are used in obtaining our generalizations.

For notation and definitions not given in this paper the reader is referred to Clifford and Preston [1] and [2].

\section{Preliminary results}

Following Munn [10], let $d$ be a positive integer and let $\left\{G_{i}: i=0,1, \cdots\right.$, $d-1\}$ be a set of pairwise disjoint groups. Let $\gamma_{d-1}$ be a homomorphism of $G_{d-1}$ into $G_{0}$ and let $\gamma_{i}$ be a homomorphism of $G_{i}$ into $G_{i+1}(i=0,1, \cdots, d-2)$. Thus

1) This research was carried out at Monash University while the author held a Commonwealth Postgraduate Award. 
we have a sequence

$$
G_{0} \stackrel{\gamma_{0}}{\longrightarrow} G_{1} \stackrel{\gamma_{1}}{\longrightarrow} \cdots \stackrel{\gamma_{d-2}}{\longrightarrow} G_{d-1} \stackrel{\gamma_{d-1}}{\longrightarrow} G_{0} .
$$

Denote by $N$ the set of non-negative integers. For $n \in N$ denote by $n(\bmod d)$ the integer equivalent to $n$ modulo $d$, belonging to $N$, and less than $d$. Define $\gamma_{n}=\gamma_{n(\bmod d)}$ for $n \in N$. For $(m, n) \in N \times N$ and $m<n$ write

$$
\alpha_{m, n}=\gamma_{m} \gamma_{m+1} \cdots \gamma_{n-1},
$$

and for all $n \in N$ let $\alpha_{n, n}$ denote the identity automorphism of $G_{n(\bmod d)}$. Let $S$ be the set of all ordered triples $\left(m, a_{i}, n\right)$, where $m, n \in N, 0 \leqq i \leqq d-1$ and $a_{i} \in G_{i}$. Define a multiplication in $S$ as follows:

$$
\left(m, a_{i}, n\right) \cdot\left(p, b_{j}, q\right)=\left(m+p-p \wedge n,\left(a_{i} \alpha_{u, w}\right)\left(b_{j} \alpha_{v, w}\right), q+n-p \wedge n\right),
$$

where $p \wedge n=\min \{p, n\}, u=n d+i, v=p d+j$ and $w=\max \{u, v\}$. Denote the groupoid so formed by $S\left(d ; G_{0}, \cdots, G_{d-1} ; \gamma_{0}, \cdots, \gamma_{d-1}\right)$ or, more compactly, by $S\left(d ; G_{i} ; \gamma_{i}\right)$. Then, as was shown in $[10], S\left(d ; G_{i} ; \gamma_{i}\right)$ is a simple regular $\omega$ semigroup and any simple regular $\omega$-semigroup is isomorphic to a semigroup $S\left(d ; G_{i} ; \gamma_{i}\right)$.

For $0 \leqq i \leqq d-1$ put $S_{i}=\left\{\left(m, a_{i}, n\right): m, n \in N, a_{i} \in G_{i}\right\} . S_{i}$ is a bisimple subsemigroup of $S$; further

$$
S=\bigcup_{0 \leqq i \leqq d-1} S_{i}
$$

It is evident that $\alpha_{i, i+d}$ is an endomorphism of $G_{i}$. In the terminology of Reilly [11], $S_{i}=S_{i}\left(G_{i}, \alpha_{i, i+d}\right)$.

For $n \in N$ and $i=0,1, \cdots, d-1$ write $e_{i}^{n}=\left(n, e_{i}, n\right)$, where $e_{i}$ is the identity of the group $G_{i}$. The elements $e_{i}^{n}$ are the idempotents of $S\left(d ; G_{i} ; \gamma_{i}\right)$ and we have

$$
e_{0}^{0}>e_{1}^{0}>\cdots>e_{d-1}^{0}>e_{0}^{1}>e_{1}^{1}>\cdots>e_{d-1}^{1}>e_{0}^{2}>\cdots .
$$

The semigroup $S\left(d ; G_{i} ; \gamma_{i}\right)$ is in fact an inverse semigroup with identity $e_{0}^{0}$. Further, $\left(m, a_{i}, n\right)^{-1}=\left(n, a_{i}^{-1}, m\right)$.

Put $B=\left\{\left(m, e_{i}, n\right): m, n \in N, 0 \leqq i \leqq d-1\right\} . \quad B$ is a subsemigroup of $S\left(d ; G_{i}, \gamma_{i}\right)$. We note that $B$ is uniquely determined by the number $d$. When $d=1, B$ becomes the bicyclic semigroup.

A congruence $\rho$ on a semigroup $S$ is called idempotent-separating if each congruence class contains at most one idempotent of $S$. Lallement [5] has proved that a congruence on a regular semigroup is idempotent-separating if and only if it is contained in Green's equivalence $\mathscr{H}$. From the definition of multiplication in $S\left(d ; G_{i} ; \gamma_{i}\right)$ it is easy to show that the equivalence $\mathscr{H}$ on a simple regular $\omega$ semigroup is given by:

$$
\left(\left(m, a_{i}, n\right),\left(p, b_{j}, q\right)\right) \in \mathscr{H} \text { if and only if } i=j, m=p \text { and } n=q .
$$

This result will be used frequently. In fact $\mathscr{H}$ is a congruence, as we now prove. 
Lemma 1.1. (cf. Munn [10], Theorem 2.1). Let $S=S\left(d ; G_{i} ; \gamma_{i}\right)$. Then $\mathscr{H}$ is a congruence on $S$ and $S / \mathscr{H} \cong B$.

Proof. The mapping $\theta$ of $S$ onto $B$ defined by $\left(m, a_{i}, n\right) \theta=\left(m, e_{i}, n\right)$ is a homomorphism. Further $\left(\left(m, a_{i}, n\right),\left(p, b_{j}, q\right)\right) \in \mathscr{H}$ if and only if $\left(m, e_{i}, n\right)=$ $\left(p, e_{j}, q\right)$; hence $\theta \circ \theta^{-1}=\mathscr{H}$ and the result follows.

A congruence $\rho$ on a semigroup $S$ is called a group congruence if $S / \rho$ is a group. The following lemma provides a characterization of the minimum group congruence $\sigma$ on an inverse semigroup.

LemMa 1.2. (Munn [6], Theorem 1). Let $S$ be an inverse semigroup and let a relation $\sigma$ be defined on $S$ by the rule that $(x, y) \in \sigma$ if and only if $e x=e y$ for some idempotent $e$ in $S$ (or, equivalently, if and only if $x f=y f$ for some idempotent $f$ in $S$ ). Then $\sigma$ is a group congruence on $S$. Furthermore, a congruence $\rho$ on $S$ is a group congruence if and only if $\sigma \subseteq \rho$ and so $S / \rho$ is isomorphic with some quotient group of $S / \sigma$.

Let $\Lambda_{0}(S)$ denote the set of congruences on a simple regular $\omega$-semigroup $S$ consisting of those congruences which either are idempotent-separating or are group congruences on $S$.

Evidently $\Lambda_{0}(S)$ is a sublattice of the lattice $\Delta(S)$ of all congruences on $S$. For $\alpha, \beta \in \Lambda(S)$ and $\alpha \subseteq \beta$, define

$$
[\alpha, \beta]=\{\lambda \in \Lambda(S): \alpha \subseteq \lambda \subseteq \beta\} ;
$$

$[\alpha, \beta]$ is a sublattice of $\Lambda(S)$. Then $[i, \mathscr{H}]$ is the set of all idempotent-separating congruences on $S$ and $[\sigma, S \times S]$ is the set of all group congruences on $S$. Thus

$$
\Lambda_{0}(S)=[i, \mathscr{H}] \cup[\sigma, S \times S] .
$$

A lattice $\Pi$ is modular if and only if for any elements $\alpha, \beta, \gamma \in \Pi$,

$$
\alpha \leqq \beta \text { implies } \alpha \vee(\beta \wedge \gamma)=\beta \wedge(\alpha \vee \gamma) .
$$

It is well known that the lattice of normal subgroups of a group is modular (see for instance Hall [3]); hence [ $\sigma, S \times S]$ is modular since it is isomorphic to the lattice of congruences on the group $S / \sigma$. Munn [7] has proved that for regular semigroups the lattice of all congruences contained in Green's equivalence $\mathscr{H}$ is modular. Hence $[i, \mathscr{H}]$ is modular,

\section{Idempotent-separating congruences}

Let $S=S\left(d ; G_{i} ; \gamma_{i}\right)$ be a simple regular $\omega$-semigroup. Put $G=G_{0} \times G_{1} \times$ $\cdots \times G_{d-1}$, the cartesian product of $G_{i}, i=0,1, \cdots, d-1$. A subset $A$ of $G$ will be called $\gamma$-admissible if it satisfies the following three conditions: 
(i) $A=A_{0} \times A_{1} \times \cdots \times A_{d-1}$, for some $A_{i} \subseteq G_{i}, i=0,1, \cdots, d-1$,

(ii) $A_{i} \otimes G_{i}$, for $i=0,1, \cdots, d-1$, and

(iii) $A_{d-1} \gamma_{d-1} \subseteq A_{0}$ and $A_{i} \gamma_{i} \subseteq A_{i+1}$, for $i=0,1, \cdots, d-2$.

A subset $A$ of $G$ will be called normal if it satisfies (i) and (ii) above.

We have already noted that $\alpha_{i, i+d}$ is an endomorphism of $G_{i}$, for $i=0$, $1, \cdots, d-1$. Munn [9] defines a subgroup $A_{i}$ of $G_{i}$ to be $\alpha_{i, i+d^{-}}$admissible if (i) $A_{i} \preccurlyeq G_{i}$ and (ii) $A_{i} \alpha_{i, i+d} \subseteq A_{i}$. Suppose that $A=A_{0} \times A_{1} \times \cdots \times A_{d-1}$ is a $\gamma$-admissible subset of $G$. Then it is easily verified that $A_{i}$ is $\alpha_{i, i+d}$-admissible for $i=0,1, \cdots, d-1$.

If $A=A_{0} \times A_{1} \times \cdots \times A_{d-1}$ and $B=B_{0} \times B_{1} \times \cdots \times B_{d-1}$ are normal subsets of $G$, we define

$$
\begin{aligned}
& A \vee B=A_{0} B_{0} \times A_{1} B_{1} \times \cdots \times A_{d-1} B_{d-1} \text { and } \\
& A \wedge B=A_{0} \cap B_{0} \times A_{1} \cap B_{1} \times \cdots \times A_{d-1} \cap B_{d-1} .
\end{aligned}
$$

We shall often write $A B=A \vee B$. It is clear that $A B$ and $A \wedge B$ are again normal subsets of $G$. Let $\Gamma$ denote the set of $\gamma$-admissible subsets of $G$. It is easily checked that the property (iii) above is preserved under the operations of union $(\vee)$ and intersection $(\wedge)$. Thus $\Gamma$ is a sublattice of the lattice of normal subsets of $G$.

Since the lattice of normal subgroups of a group is modular it follows that the lattice of normal subsets of $G$ is modular, since the direct product of modular lattices is modular. Further, a sublattice of a modular lattice is modular and hence the lattice $\Gamma$ of $\gamma$-admissible subsets of $G$ is modular.

For any congruence $\lambda$ on $S$ we define a subset $A^{\lambda}$ of $G$ as follows:

$$
\begin{aligned}
& A^{\lambda}=A_{0}^{\lambda} \times A_{1}^{\lambda} \times \cdots \times A_{d-1}^{\lambda}, \quad \text { where } \\
& A_{i}^{\lambda}=\left\{a_{i} \in G_{i}:\left(0, a_{i}, 0\right) \in e_{i}^{0} \lambda\right\}, i=0,1, \cdots, d-1 .
\end{aligned}
$$

Evidently $A^{\lambda}=A^{\lambda \wedge \rtimes}$, since the $\mathscr{H}$-class containing $e_{i}^{0}$ is $\left\{\left(0, a_{i}, 0\right) \in S: a_{i} \in G_{i}\right\}$.

LeMma 2.1. For any congruence $\lambda$ on $S\left(d ; G_{i} ; \lambda_{i}\right) A^{\lambda}$ is a $\gamma$-admissible subset of $G$.

Proor. Put $H_{i}=\left\{\left(0, a_{i}, 0\right) \in S: a_{i} \in G_{i}\right\} ; H_{i}$ is a subgroup of $S$. Set $\lambda_{i}=\lambda \cap\left(H_{i} \times H_{i}\right)$. Then $\lambda_{i}$ is a congruence on $H_{i}$ and so $e_{i}^{0} \lambda$ is a normal subgroup of $H_{i}$. Now $G_{i}$ is isomorphic to $H_{i}$ under the mapping $a_{i} \rightarrow\left(0, a_{i}, 0\right)$ and it follows that $A_{i}^{\lambda}$ is a normal subgroup of $G_{i}$. Thus $A_{i}^{\lambda}$ is a normal subgroup of $G_{i}(i=0$, $1, \cdots, d-1)$, since our argument is independent of $i$.

Suppose $a_{i} \in A_{i}^{\lambda}$ and assume $i \neq d-1$. Put $x=\left(0, a_{i}, 0\right)$. Then $\left(x, e_{i}^{0}\right) \in \lambda$ and so

that is,

$$
\left(x e_{i+1}^{0}, e_{i}^{0} e_{i+1}^{0}\right) \in \lambda,
$$

$$
\left(\left(0, a_{i} \gamma_{i}, 0\right), e_{i+1}^{0}\right) \in \lambda
$$


hence $a_{i} \gamma_{i} \in A_{i+1}^{\lambda}$. On the other hand, if $i=d-1$, put $x=\left(0, a_{d-1}, 0\right)$ and $y=\left(0, e_{0}, 1\right)$. Then $\left(x, e_{d-1}^{0}\right) \in \lambda$ and so

But

$$
\left(y x y^{-1}, y e_{d-1}^{0} y^{-1}\right) \in \lambda .
$$

$$
y x y^{-1}=\left(0, a_{d-1} \gamma_{d-1}, 0\right)
$$

and $y e_{d-1}^{0} y^{-1}=e_{0}^{0}$. Hence $a_{d-1} \gamma_{d-1} \in A_{0}^{\lambda}$. Thus $A^{\lambda}$ is $\gamma$-admissible. Then

LEMMA 2.2. (i) Let $\lambda$ be an idempotent-separating congruence on $S\left(d ; G_{i} ; \lambda_{i}\right)$.

$\left(\left(m, a_{i}, n\right),\left(p, b_{j}, q\right)\right) \in \lambda$ if and only if $i=j, m=p, n=q$ and $a_{i} b_{j}^{-1} \in A_{i}^{\lambda}$. relation

(ii) Let $A=A_{0} \times A_{1} \times \cdots \times A_{d-1}$ be a $\gamma$-admissible subset of $G$. Then the

$$
\lambda=\left\{\left(\left(m, a_{i}, n\right),\left(p, b_{j}, q\right)\right) \in S \times S: i=j, m=p, n=q \text { and } a_{i} b_{j}^{-1} \in A_{i}\right\}
$$

is an idempotent-separating congruence on $S\left(d ; G_{i} ; \gamma_{i}\right)$. Furthermore, $A^{\lambda}=A$.

The proof of lemma 2.2 is a straightforward computation and is omitted. It follows that there exists a one-to-one correspondence between the idempotentseparating congruences of $S$ and the $\gamma$-admissible subsets of $G$. Furthermore, $\lambda \subseteq \lambda^{\prime}$ if and only if $A^{\lambda} \subseteq A^{\lambda^{\prime}}$.

By the above remark the mapping $\phi:[i, \mathscr{H}] \rightarrow \Gamma$ given by $\lambda \phi=A^{\lambda}$, which is onto by lemma 2.2 (ii), is a lattice isomorphism. This affords us a direct proof of the fact that $[i, \mathscr{H}]$ is modular, since we have noted that $\Gamma$ is a modular lattice.

Let $A$ be a $\gamma$-admissible subset of $G$. We define a mapping $\gamma_{d-1} \mid A$ of $G_{d-1} /$ $A_{d-1}$ into $G_{0} / A_{0}$ as follows:

$$
\left(a_{d-1} A_{d-1}\right)\left(\gamma_{d-1} \mid A\right)=\left(a_{d-1} \gamma_{d-1}\right) A_{0}, \text { for all } a_{d-1} A_{d-1} \in G_{d-1} / A_{d-1} .
$$

Further, for $i=0,1, \cdots, d-2$, we define a mapping $\gamma_{i} \mid A$ of $G_{i} / A_{i}$ into $G_{i+1} / A_{i+1}$ as follows:

$$
\left(a_{i} A_{i}\right)\left(\gamma_{i} \mid A\right)=\left(a_{i} \gamma_{i}\right) A_{i+1}, \text { for all } a_{i} A_{i} \in G_{i} / A_{i} .
$$

That these mappings are well defined is a consequence of the $\gamma$-admissibility of $A$. It is immediate that $\gamma_{i} \mid A$ is a homomorphism for $i=0,1, \cdots, d-1$. For $n \in N$ define $\gamma_{n}\left|A=\gamma_{n(\bmod d)}\right| A$. For $(m, n) \in N \times N$ and $m<n$ write

$$
\alpha_{m, n} \mid A=\left(\gamma_{m} \mid A\right)\left(\gamma_{m+1} \mid A\right) \cdots\left(\gamma_{n-1} \mid A\right),
$$

and for all $n \in N$ let $\alpha_{n, n} \mid A$ denote the identity automorphism of $G_{n(\bmod d)} / A_{n(\bmod d)}$. Further, if we assume that $m=p d+i$ and $n=q d+j$, then

$$
\left(a_{i} A_{i}\right)\left(\alpha_{m, n} \mid A\right)=\left(a_{i} \alpha_{m, n}\right) A_{j} \text {. }
$$

THEOREM 2.1. Let $\lambda$ be an idempotent-separating congruence on $S\left(d ; G_{i} ; \gamma_{i}\right)$. Then

$$
S / \lambda \cong S\left(d ; G_{i} / A_{i}^{\lambda} ; \gamma_{i} \mid A^{\lambda}\right)
$$


Proof. Consider the mapping $\theta$ of $S$ onto $S\left(d ; G_{i} / A_{i}^{\lambda} ; \gamma_{i} \mid A^{\lambda}\right)$ defined by

$$
\left(m, a_{i}, n\right) \theta=\left(m, a_{i} A_{i}^{\lambda}, n\right) .
$$

Then $\theta$ is a homomorphism. Further $\left(m, a_{i}, n\right) \theta=\left(p, b_{j}, q\right) \theta$ if and only if $i=j, m=p, n=q$ and $a_{i} A_{i}^{\lambda}=b_{j} A_{j}^{\lambda}$. By lemma 2.2 (i) these equations hold if and only if $\left(\left(m, a_{i}, n\right),\left(p, b_{j}, q\right)\right) \in \lambda$. Hence $\theta \circ \theta^{-1}=\lambda$ which gives the result.

Let ker $\alpha_{i, i+d}^{n}$ denote the kernel of the endomorphism $\alpha_{i, i+d}^{n}$ of $A_{i}$, for $i=0,1, \cdots, d-1$ and $n=1,2, \cdots$. Put $K_{i}^{n}=\operatorname{ker} \alpha_{i, i+d}^{n}, K_{i}=\bigcup_{n=1}^{\infty} K_{i}^{n}$ and $K=K_{0} \times K_{1} \times \cdots \times K_{d-1}$.

LEMMA 2.3. Let $\sigma$ be the minimal group congruence on $S=S\left(d ; G_{i} ; \gamma_{i}\right)$. Then $A^{\sigma \wedge \mathscr{*}}=A^{\sigma}=K$.

Proof. We have noted earlier that $A^{\sigma \wedge}=A^{\sigma}$. Let $a_{i} \in A_{i}^{\sigma}$. Then $\left(\left(0, a_{i}, 0\right)\right.$, $\left.e_{i}^{0}\right) \in \sigma$ and so, by Lemma 1.2 , there exists $e_{j}^{m}$ such that $e_{j}^{m}\left(0, a_{i}, 0\right)=e_{j}^{m} e_{i}^{0}$. We may suppose without loss of generality that $m>0$. Hence $\left(m, a_{i} \alpha_{i, m d+j}, m\right)=$ $e_{j}^{m}$ and so $a_{i} \alpha_{i, m d+j}=e_{j}$; it follows that $\left(a_{i} \alpha_{i, m d+j}\right) \alpha_{j, d+i}=e_{i}$. Thus $a_{i} \in K_{i}$.

Conversely, let $a_{i} \in K_{i}$. Then $a_{i} \alpha_{i, i+d}^{m}=e_{i}$ for some $m$, and so $e_{i}^{m}\left(0, a_{i}, 0\right)=$ $e_{i}^{m} e_{i}^{0}$. Hence, by lemma $1.2,\left(\left(0, a_{i}, 0\right), e_{i}^{0}\right) \in \sigma$; that is, $a_{i} \in A_{i}^{\sigma}$. We conclude that $A_{i}^{\sigma}=K_{i}(i=0,1, \cdots, d-1)$, and the result follows.

Corollary 2.1. Let $S=S\left(d ; G_{i} ; \gamma_{i}\right)$. Then

$$
S / \sigma \wedge \mathscr{H} \cong S\left(d ; G_{i} / K_{i}: \gamma_{i} \mid K\right) \text {. }
$$

Corollary 2.1 follows immediately from theorem 2.1 .

\section{Group congruences}

We begin this section with a general result about inverse semigroups with identity.

LeMma 3.1. (Munn [9], Lemma 4 (ii)). Let $\rho$ be a group congruence on an inverse semigroup $S$ with identity $e$. Then $(x, y) \in \rho$ if and only if $x y^{-1} \in e \rho$.

Let $A=A_{0} \times A_{1} \times \cdots \times A_{d-1}$ be a $\gamma$-admissible subset of $G$. Then, as was

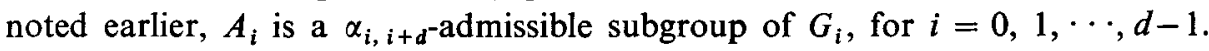
Following Munn [9], we define $\operatorname{rad} A_{i}$, the radical of $A_{i}$ relative to the endomorphism $\alpha_{i, i+d}$, as follows:

$$
\operatorname{rad} A_{i}=\left\{a_{i} \in G_{i}: a_{i} \alpha_{i, i+d}^{n} \in A_{i} \text { for some } n\right\} .
$$

Using these radicals we define the radical of $A, \operatorname{Rad} A$, as follows:

$$
\operatorname{Rad} A=\operatorname{rad} A_{0} \times \operatorname{rad} A_{1} \times \cdots \times \operatorname{rad} A_{d-1} .
$$

$\operatorname{Rad} A$ is a normal subset of $G$, since each $\operatorname{rad} A_{i} \ngtr G_{i}$ (Munn [9]). In fact $\operatorname{Rad} A$ is a $\gamma$-admissible subset of $G$, as we now show. Let $a_{i} \in \operatorname{rad} A_{i}$, that is, let 
and so

$$
a_{i} \alpha_{i, i+d}^{n} \in A_{i} \text { for some } n,
$$

But

$$
\left(a_{i} \alpha_{i, i+d}^{n}\right) \gamma_{i} \in A_{i+1(\bmod d)}
$$

Thus $\operatorname{Rad} A \in \Gamma$.

$$
\left(a_{i} \alpha_{i, i+d}^{n}\right) \gamma_{i}=\left(a_{i} \gamma_{i}\right) \alpha_{i+1, i+1+d}^{n}
$$

Again, directly generalizing Munn's procedure in [9], we denote the $\gamma$-admissible set $\left\{\left(e_{0}, e_{1}, \cdots, e_{d-1}\right)\right\}$ by 1 . Then $\operatorname{Rad} 1=K$. Hence it follows that $A^{\sigma}=\operatorname{Rad} 1$. Put $\Gamma^{*}=\{A \in \Gamma: \operatorname{Rad} A=A\}$.

That the following properties hold for Rad follows immediately from Munn's lemma 2 in [9], where it is shown that the analogous properties hold for rad in each component $G_{i}$ of $G$.

(i) $A \subseteq \operatorname{Rad} A$,

(ii) $A \subseteq A^{\prime}$ implies that $\operatorname{Rad} A \subseteq \operatorname{Rad} A^{\prime}$,

(iii) $\operatorname{Rad}(\operatorname{Rad} A)=\operatorname{Rad} A$,

(iv) $A \operatorname{Rad} 1 \subseteq \operatorname{Rad} A$,

(v) $\operatorname{Rad}(A \operatorname{Rad} 1)=\operatorname{Rad} A$.

Properties (i), (ii) and (iii) imply that Rad is a closure operator on the set of $\gamma$-admissible subsets of $G$.

The next lemma follows from and generalizes Munn's lemma 4 (i) in [9].

Lemma 3.2. Let $\rho \in[\sigma, S \times S]$. Then $A^{\rho} \in \Gamma^{*}$.

Proof. $\rho \mid S_{i}$ is a group congruence on $S_{i}$. Hence $\operatorname{rad} A_{i}^{\rho}=A_{i}^{\rho}$ by Munn's lemma 4 (i) in [9]. Thus $\operatorname{Rad} A^{\rho}=A^{\rho}$ and $A^{\rho} \in \Gamma^{*}$ as required.

We now fix our attention on the sublattice $[\sigma, \sigma \vee \mathscr{H}]$, and begin by determining the congruence $\sigma \vee \mathscr{H}$. Note that the restriction to each $S_{i}$ gives Munn and Reilly's determination of $\sigma \vee \mathscr{H}$ on a bisimple $\omega$-semigroup [8].

Lemma 3.3. Let $S=S\left(d ; G_{i} ; \gamma_{i}\right)$. Then

$$
\left(\left(m, a_{i}, n\right),\left(p, b_{j}, q\right)\right) \in \sigma \vee \mathscr{H} \text { if and only if } m-n=p-q .
$$

Proof. Let $x=\left(m, a_{i}, n\right)$ and $y=\left(p, b_{j}, q\right)$. Suppose that $(x, y) \in \sigma \vee \mathscr{H}$. Then since $\sigma \vee \mathscr{H}=\sigma \circ \mathscr{H} \circ \sigma$ (Howie [4], Theorem 3.9) there exist $u, v$ in $S$ such that $(x, u) \in \sigma,(u, v) \in \mathscr{H}$, and $(v, y) \in \sigma$. Let $u=\left(m^{\prime}, g_{k}, n^{\prime}\right)$ and $v=$ $\left(p^{\prime}, h_{t}, q^{\prime}\right)$. Since $(x, u) \in \sigma$ there exists, by lemma 1.2, an idempotent $e_{s}^{r}$ such that $e_{s}^{r} x=e_{s}^{r} u$, and we can assume without loss of generality that $r \geqq m, m^{\prime}$. Hence we have that $r+n-m=r+n^{\prime}-m^{\prime}$ and so $m-n=m^{\prime}-n^{\prime}$. Similarly, since $(v, y) \in \sigma$ we have $p-q=p^{\prime}-q^{\prime}$. But $m^{\prime}-p^{\prime}=n^{\prime}-q^{\prime}$ since $(u, v) \in \mathscr{H}$. Hence $m-n=p-q$.

Conversely, let $x$ and $y$ be such that $m-n=p-q$. We may assume that $m \leqq p$. Suppose $m<p$ or $m=p$ and $j \geqq i$. Then 


$$
\begin{aligned}
e_{j}^{p} x & =\left(p, e_{j}, p\right)\left(m, a_{i}, n\right) \\
& =\left(p, a_{i} \alpha_{m d+i, p d+j}, p+n-m\right) \\
& =\left(p, a_{i} \alpha_{m d+i, p d+j}, q\right)
\end{aligned}
$$

and so $\left(e_{j}^{p} x, y\right) \in \mathscr{H}$. But $\left(x, e_{j}^{p} x\right) \in \sigma$ since $e_{j}^{p}$ is idempotent. Hence $(x, y) \in \sigma \circ \mathscr{H}$ $\subseteq \sigma \vee \mathscr{H}$

Suppose now that $m=p$ and $j<i$. Then

$$
\begin{aligned}
e_{i}^{p} y & =\left(p, e_{i}, p\right)\left(p, b_{j}, q\right) \\
& =\left(p, b \alpha_{p d+j, p d+i}, q\right)
\end{aligned}
$$

and so $\left(e_{i}^{p} y, x\right) \in \mathscr{H}$. But $\left(y, e_{i}^{p} y\right) \in \sigma$ since $e_{i}^{p}$ is idempotent. Hence $(x, y) \in \sigma \circ$ $\mathscr{H} \subseteq \sigma \vee \mathscr{H}$.

COROLLARY 3.1. $S / \sigma \vee \mathscr{H} \cong Z$, where $Z$ denotes the integers.

Proof. Consider the mapping $\theta: S \rightarrow Z$ defined by $\left(m, a_{i}, n\right) \theta=m-n$. The mapping $\theta$ is a homomorphism and it follows that $\theta \circ \theta^{-1}=\sigma \vee \mathscr{H}$.

LeMma 3.4. (i) Let $\rho \in[\sigma, \sigma \vee \mathscr{H}]$. Then

$$
e_{0}^{0} \rho=\left\{\left(m, a_{i}, m\right) \in S: m \in N, a_{i} \in A_{i}, 0 \leqq i \leqq d-1\right\} .
$$

(ii) Let $\rho, \rho^{\prime} \in[\sigma, \sigma \vee \mathscr{H}]$. Then $\rho \subseteq \rho^{\prime}$ if and only if $A^{\rho} \subseteq A^{\rho^{\prime}}$.

Proof. (i) Since $\rho$ is a group congruence, $\left(e_{0}^{0}, e_{i}^{0}\right) \in \rho$ for $0 \leqq i \leqq d-1$. Hence

$$
e_{0}^{0} \rho=\bigcup_{0 \leqq i \leqq d-1} e_{i}^{0}\left(\rho \mid S_{i}\right)
$$

and our result follows from Munn [9] lemma 5(i).

(ii) If $\rho \subseteq \rho^{\prime}$ then clearly $A^{\rho} \subseteq A^{\rho^{\prime}}$. Suppose conversely that $A^{\rho} \subseteq A^{\rho^{\prime}}$. Then by (i), $e_{0}^{0} \rho \subseteq e_{0}^{0} \rho^{\prime}$. Let $(x, y) \in \rho$. Then $x y^{-1} \in e_{0}^{0} \rho$ by lemma 3.1. Hence $x y^{-1} \in e_{0}^{0} \rho^{\prime}$ and so $(x, y) \in \rho$, again by lemma 3.1. We conclude that $\rho \subseteq \rho^{\prime}$.

We now prove a partial converse to lemma 3.2 which generalizes Munn's lemma 5 (iii) in [9].

Lemma 3.5. Let $A \in \Gamma^{*}$. Then there exists $\tau$ in $[\sigma, \sigma \vee \mathscr{H}]$ such that $A^{\tau}=A$.

Proof. For $x=\left(m, a_{i}, n\right)$ and $y=\left(p, b_{j}, q\right)$ in $S$ write

$$
\|x y\|=a_{i} \alpha_{u, w} b_{j}^{-1} \alpha_{v, w},
$$

where $u=n d+i, v=q d+j$ and $w=\max \{u, v\}$. Define

$$
\begin{aligned}
\tau=\left\{(x, y) \in S \times S: x=\left(m, a_{i}, n\right), y\right. & =\left(p, b_{j}, q\right), \\
m-n & \left.=p-q \text { and }\|x y\| \in A_{k} \text { for some } k\right\} .
\end{aligned}
$$


We shall show that $\tau$ is a congruence on $S$ with the desired property. It is straightforward to show that $\tau$ is reflexive and symmetric.

Let now $x=\left(m, a_{i}, n\right), y=\left(p, b_{j}, q\right)$ and $z=\left(r, c_{k}, s\right)$. Suppose $(x, y) \in \tau$ and $(y, z) \in \tau$. Then $m-n=p-q$ and $p-q=r-s$ and so $m-n=r-s$. To prove that $\tau$ is transitive it remains to show that $\|x z\| \in A_{t}$, for some $t$. We may assume without loss of generality that $n<s$ or $n=s$ and $i \leqq k$. We now proceed by cases.

CASE (i): $q<n$ or $q=n$ and $j \leqq i$.

Then $\quad\|x z\|=a_{i} \alpha_{n d+i, s d+k} c_{k}^{-1}$

$$
\begin{aligned}
& =a_{i} \alpha_{n d+i, s d+k}\left(b_{j}^{-1} b_{j}\right) \alpha_{q d+j, s d+k} c_{k}^{-1} \\
& =\left(a_{i} \alpha_{n d+i, s d+k} b_{j}^{-1} \alpha_{q d+j, s d+k}\right)\left(b_{j} \alpha_{q d+j, s d+k} c_{k}^{-1}\right) \\
& =\left(a_{i} \alpha_{n d+i, s d+k}\left(b_{j}^{-1} \alpha_{q d+j, n d+i}\right) \alpha_{n d+i, s d+k}\right)\left(b_{j} \alpha_{q d+j, s d+k} c_{k}^{-1}\right) \\
& =\left(a_{i} b_{j}^{-1} \alpha_{q d+j, n d+i}\right) \alpha_{n d+i, s d+k}\left(b_{j} \alpha_{q d+j, s d+k} c_{k}^{-1}\right) \\
& =\|x y\| \alpha_{n d+i, s d+k}\|y z\| \\
& \in A_{k}, \text { since }\|x y\| \in A_{i},\|y z\| \in A_{k} \text { and } A \text { is } \gamma \text {-admissible. }
\end{aligned}
$$

Hence $(x, z) \in \tau$.

CASE (ii): $n<q<s$ or $n=q$ and $i \leqq j$ or $q=s$ and $j \leqq k$.

Then $\quad\|x z\|=a_{i} \alpha_{n d+i, s d+k} c_{k}^{-1}$

$$
\begin{aligned}
& =a_{i} \alpha_{n d+i, s d+k}\left(b_{j} b_{j}^{-1}\right) \alpha_{q d+j, s d+k} c_{k}^{-1} \\
& =\left(a_{i} \alpha_{n d+i, q d+j}\right) \alpha_{q d+j, s d+k} b_{j} \alpha_{q d+j, s d+k} b_{j}^{-1} \alpha_{q d+j, s d+k} c_{k}^{-1} \\
& =\left(a_{i} \alpha_{n d+i, q d+j} b_{j}^{-1}\right) \alpha_{q d+j, s d+k} b_{j} \alpha_{q d+j, s d+k} c_{k}^{-1} \\
& =\|x y\| \alpha_{q d+j, s d+k}\|y z\| \\
& \in A_{k}, \text { since }\|x y\| \in A_{j},\|y z\| \in A_{k} \text { and } A \text { is } \gamma \text {-admissible. }
\end{aligned}
$$

Hence $(x, z) \in \tau$.

CASE (iii): $s<q$ or $s=q$ and $k \leqq j$.

Then

$$
\begin{aligned}
\|x z\| \alpha_{s d+k, q d+j} & =\left(a_{i} \alpha_{n d+i, s d+k} c_{k}^{-1}\right) \alpha_{s d+k, q d+j} \\
& =a_{i} \alpha_{n d+i, s d+k} \alpha_{s d+k, q d+j} c_{k}^{-1} \alpha_{s d+k, q d+j} \\
& =a_{i} \alpha_{n d+i, q d+j} b_{j}^{-1} b_{j} c_{k}^{-1} \alpha_{s d+k, q d+j} \\
& =\|x y\|\|y z\| \\
& \in A_{j}, \text { since }\|x y\| \in A_{j} \text { and }\|y z\| \in A_{j} .
\end{aligned}
$$

Hence $\|x z\| \alpha_{s d+k, q d+j} \alpha_{j+d, k} \in A_{k}$, that is $\|x z\| \alpha_{k, k+d}^{q-s+1} \in A_{k}$. Now $\operatorname{Rad} A=A$ and so $\|x z\| \in A_{k}$. Hence $(x, z) \in \tau$. We conclude that $\tau$ is an equivalence relation on $\mathrm{S}$.

That $\tau$ is left and right compatible follows from computations similar to those above and they are left to the reader. 
It follows from lemma 3.3 and the definition of $\tau$ that $\tau \in[\sigma, \sigma \vee \mathscr{H}]$. It also follows from the definition of $\tau$ that $A^{\tau}=A$.

We note here that Munn's proof of lemma 5 (iii) in [9] can be generalized to give an alternative proof of our lemma 3.5 by making use of the closed selfconjugate subsemigroup of $S$.

$$
M=\left\{\left(m, a_{i}, m\right): m \in N, a_{i} \in A_{i}, 0 \leqq i \leqq d-1\right\}
$$

Then $\tau=\left\{(x, y) \in S \times S: x y^{-1} \in M\right\}$ is a congruence on $S$ and $A=A^{\tau}$.

Partially ordering $\Gamma^{*}$ by inclusion we obtain from Lemma 3.5 and lemma 3.4 (ii) that

$$
\Gamma^{*} \cong\left[\sigma, \sigma \vee \mathscr{H}^{*}\right] \text {. }
$$

Now $[\sigma, \sigma \vee \mathscr{H}]$ is a modular lattice since it is a sublattice of the modular lattice $[\sigma, S \times S]$. We conclude that $\Gamma^{*}$ is a modular lattice.

\section{The main result}

We are now in a position to generalize Munn's argument to the present situation and prove an analogue of his main theorem in [9], viz.

THEOREM 4.1. Let $S$ be a simple regular $\omega$-semigroup. Then the sublattice $\Lambda_{0}(S)$ is modular, if and only if $\operatorname{Rad} A=A \operatorname{Rad} 1$ for all $A$ in $\Gamma$.

To establish this result we establish the analogues of lemmas 8,9 and 10 of [9]. To do this we need two preliminary lemmas which generalize Munn's lemmas 6 and 7 of [9].

Lemma 4.1. Let $\lambda \in[i, \mathscr{H}]$ and $\rho \in[\sigma, \sigma \vee \mathscr{H}]$. Then

(i) $\lambda \vee \rho \in[\sigma, \sigma \vee \mathscr{H}]$ and $A=\operatorname{Rad}\left(A^{\lambda} A^{\rho}\right)$, and

(ii) $\lambda \wedge \rho \in[i, \mathscr{H}]$ and $A^{\lambda \wedge \rho}=A^{\lambda} \wedge A^{\rho}$.

Lemma 4.2. $A^{\lambda \vee \sigma}=\operatorname{Rad} A^{\lambda}$ for any $\lambda$ in $[i, \mathscr{H}]$.

To prove lemma 4.1 and 4.2 it suffices to note that Munn's argument in lemma 6 and lemma 7 can be applied in a co-ordinatewise fashion to the present situation.

The next three lemmas are the analogues of lemmas 8, 9 and 10 of [9].

Lemma 4.3. Let $A_{0}(S)$ be modular and let $A \in \Gamma$. Then $\operatorname{Rad} A=A \operatorname{Rad} 1$.

Lemma 4.4. Let $\operatorname{Rad} A=A \operatorname{Rad} 1$ for all $A \in \Gamma$. Then $[i, \mathscr{H}] \cup[\sigma, \sigma \vee \mathscr{H}]$ is modular.

Lemma 4.5. Let $[i, \mathscr{H}] \cup[\sigma, \sigma \vee \mathscr{H}]$ be modular. Then $\Lambda_{0}(S)$ is modular.

To prove lemmas 4.3 and 4.4 we argue as Munn does in his lemmas 8 and 9 using the preceeding generalized lemmas. The proof of lemma 4.5 is identical to Munn's proof of lemma 10.

Theorem 4.1 now follows from lemmas 4.3, 4.4 and 4.5. 


\section{References}

[1] A. H. Clifford and G. B. Preston, The algebraic theory of semigroups, Volume I (Math. Surveys, Number 7, Amer. Math. Soc. 1961).

[2] A. H. Clifford and G. B Preston, The algebraic theory of semigroups, Volume II (Math. Surveys, Number 7, Amer. Math. Soc. 1967).

[3] M. Hall, Jnr., The theory of Groups (The Macmillan Company, New York, 1959).

[4] J. M. Howie, 'The maximum idempotent-separating congruence on an inverse semigroup', Proc. Edinburgh Math. Soc. 14 (1964), 71-79.

[5] G. Lallement, 'Congruences et équivalences de Green sur un demi-groupe régulier', $C$. $R$. Acad. Sc. Paris Série A262 (1966), 613-616.

[6] W. D. Munn, 'A class of irreducible matrix representations of an arbitraty inverse semigroup', Proc. Glasgow Math. Assoc. 5 (1961), 41-48.

[7] W. D. Munn, 'A certain sublattice of the congruences on a regular semigroup', Proc. Camb. Phil. Soc. 60 (1964), 385-391.

[8] W. D. Munn and N. R. Reilly, 'Congruences on a bisimple $\omega$-semigroup', Proc. Glasgow Math. Assoc. 7 (1966), 184-192.

[9] W. D. Munn, 'The lattice of congruences on a bisimple $\omega$-semigroup', Proc. Roy. Soc. Edinburgh 67 (1966), 175-184.

[10] W. D. Munn, 'Regular $\omega$-semigroups', Glasgow Math. J. 9 (1968), 46-66.

[11] N. R. Reilly, 'Bisimple $\omega$-semigroups', Proc. Glasgow Math. Assoc. 7 (1966), 160-167.

Department of Mathematics

Monash University 